\title{
Chang-Mundici construction of an enveloping unital lattice-group of a BL-algebra
}

\author{
Celestin Lele ${ }^{1}$ and Jean B Nganou ${ }^{2}$ \\ 1 Department of Mathematics and Computer Science, University of Dschang, Cameroon \\ celestinlele@yahoo.com \\ 2 Department of Mathematics, University of Oregon, Eugene, OR 97403 \\ nganou@uoregon.edu
}

\begin{abstract}
For any BL-algebra $L$, we construct an associated lattice ordered Abelian group $G_{L}$ that coincides with the Chang's $\ell$-group of an MV-algebra when the BL-algebra is an MValgebra. We prove that the Chang's group of the MV-center of any BL-algebra $L$ is a direct summand in $G_{L}$. We also find a direct description of the complement $\mathfrak{S}(L)$ of the Chang's group of the MV-center in terms of the filter of dense elements of $L$. Finally, we compute some examples of the group $G_{L}$.
\end{abstract}

\section{Introduction}

It is well known that the category of MV-algebras is equivalent to that of $\ell$-groups with strong unit [2]. This equivalence, which depends in large part on the natural algebraic addition of MV-algebras has been an essential tool in the study of MV-algebras. On the other hand, MV-algebras are BL-algebras whose negations are involutions. Given that a proper algebraic addition is now available in BL-algebras [5], it is natural to consider some of the constructions of MV-algebras that rely on the addition within the BL-algebras framework. Undoubtably, the Chang-Mundici's $\ell$-group of an MV-algebra is one of the most important such constructions [1, 2].

The main goal of the present work is to extend this construction to BL-algebras, by associating to each BL-algebra an $\ell$-group with strong unit. More specifically, we introduce good sequences in any BL-algebra $L$ and show that their set forms a commutative monoid $\left(M_{L},+, 0\right)$. Mimicking the general construction due to Grothendieck of an Abelian group from a commutative monoid, we obtain a lattice ordered Abelian group with strong unit $G_{L}$, which we shall refer to as the Chang's $\ell$-group of the BL-algebra. We show that the Chang's group of the MV-center of $L$ is a direct summand in $G_{L}$, and that its complement $\mathfrak{S}(L)$ is an ideal of $G_{L}$. For BL-chains, we obtain that $G_{L}$ is isomorphic to the lexicographic product of the Chang's group of the MV-center and $\mathfrak{S}(L)$. It turns out that the $\ell$-group $\mathfrak{S}(L)$ can be directly constructed from the filter of dense elements of the BL-algebra $L$, thus generalizing the relationship between BL-algebras and negative cones of $\ell$-groups 44.

\section{Addition and Good Sequences in BL-algebras}

The following natural commutative operation + was introduced in every BL-algebra [5]: For every $x, y \in L$,

$$
x+y:=(\bar{x} \rightarrow y) \wedge(\bar{y} \rightarrow x)
$$

This addition is commutative, associative, has 0 for identity, and 1 as absorbing.

Definition 2.1. A BL-chain $L$ is called of cancellative type if for every $x, y, z \in L$,

$$
x+y=x+z \text { and } x \otimes y=x \otimes z \text { imply } y=z .
$$

A BL-algebra is called of cancellative type if it is a subdirect product of BL-chains of cancellative type. 
Examples of BL-algebras of cancellative type include MV-algebras, and product algebras.

Lemma 2.2. In every BL-algebra $L$, the following equation holds.

$$
(x \otimes y)+((x+y) \otimes z)=(x \otimes z)+((x+z) \otimes y)
$$

The following definitions are motivated by similar concepts in MV-algebras.

Definition 2.3. Let $L$ be a BL-algebra. A sequence $\mathbf{a}:=\left(a_{1}, a_{2}, \ldots, a_{n}, \ldots\right)$ of elements of $L$ is called a good sequence if for all $i, a_{i}+a_{i+1}=a_{i}$, and there exists an integer $n$ such that $a_{r}=0$ for all $r>n$. In this case, instead of writing $\mathbf{a}=\left(a_{1}, a_{2}, \ldots, a_{n}, 0,0 \ldots\right)$, we will simply write $\mathbf{a}=\left(a_{1}, a_{2}, \ldots, a_{n}\right)$

Note that if, $\mathbf{a}=\left(a_{1}, a_{2}, \ldots, a_{n}\right)$ is a good sequence, then $(\underbrace{1, \ldots, 1}_{m^{\prime} s 1}, a_{1}, a_{2}, \ldots, a_{n})$ is again a good sequence. The later will be denoted by $\left(1^{m}, a_{1}, a_{2}, \cdots, a_{n}\right)$.

We define the addition of sequences as follows:

Definition 2.4. Let $L$ be a BL-algebra and let $\mathbf{a}=\left(a_{1}, a_{2}, \ldots, a_{n}, \ldots\right), \mathbf{b}=\left(b_{1}, b_{2}, \ldots, b_{n}, \ldots\right)$ be sequences in $L$. We define the sum of $\mathbf{a}$ and $\mathbf{b}$ by $\mathbf{a}+\mathbf{b}=\left(c_{1}, c_{2}, \ldots, c_{n}, \ldots\right)$ where $c_{i}:=a_{i}+\left(a_{i-1} \otimes\right.$ $\left.b_{1}\right)+\cdots+\left(a_{1} \otimes b_{i-1}\right)+b_{i}$.

Proposition 2.5. For every BL-algebra $L,\left(M_{L},+,(0)\right)$ is an Abelian monoid satisfying: for every $\mathbf{a}, \mathbf{b} \in M_{L}$,

$$
\mathbf{a}+\mathbf{b}=(0) \quad \text { implies } \quad \mathbf{a}=\mathbf{b}=(0)
$$

Using the Grothendieck construction of a group from a commutative monoid, we define on $M_{L} \times M_{L}$ the relation $\sim$ by $(\mathbf{a}, \mathbf{b}) \sim(\mathbf{c}, \mathbf{d})$ if and only if there exists $\mathbf{k} \in M_{L}$ such that $\mathbf{a}+\mathbf{d}+\mathbf{k}=\mathbf{b}+\mathbf{c}+\mathbf{k}$. Then, it is easy to see that $\sim$ is an equivalence relation on $M_{L} \times M_{L}$. Let $G_{L}=M_{L} \times M_{L} / \sim$ be the factor set of $M_{L} \times M_{L}$ by $\sim$. If we denote the equivalence class of $(\mathbf{a}, \mathbf{b})$ by $[\mathbf{a}, \mathbf{b}]$, one has a well-defined operation + on $G_{L}$ given by $[\mathbf{a}, \mathbf{b}]+[\mathbf{c}, \mathbf{d}]=[\mathbf{a}+\mathbf{c}, \mathbf{b}+\mathbf{d}]$. Moreover, $\left(G_{L},+,[(0),(0)]\right)$ is an Abelian group where $-[\mathbf{a}, \mathbf{b}]=[\mathbf{b}, \mathbf{a}]$. We define $\leq$ on the monoid $\left(M_{L},+\right)$ by: $\mathbf{a}, \mathbf{b} \in M_{L} ; \mathbf{a} \leq \mathbf{b}$ if $a_{i} \leq b_{i}$ for all $i$. It is clear that $\leq$ is a partial order on $M_{L}$.

Proposition 2.6. Let $\preceq$ be the relation defined on $G_{L}$ by $[\mathbf{a}, \mathbf{b}] \preceq[\mathbf{c}, \mathbf{d}]$ if and only if there exists $\mathbf{k} \in M_{L}$ such that $\mathbf{a}+\mathbf{d}+\mathbf{k} \leq \mathbf{b}+\mathbf{c}+\mathbf{k}$.

Then $\preceq$ is a partial order relation on $G_{L}$ that is translation invariant.

Theorem 2.7. For every BL-algebra $L$, the po-group $G_{L}$ is a lattice ordered group where,

$$
[\mathbf{a}, \mathbf{b}] \vee[\mathbf{c}, \mathbf{d}]=[(\mathbf{a}+\mathbf{d}) \vee(\mathbf{b}+\mathbf{c}), \mathbf{b}+\mathbf{d}] \quad \text { and }[\mathbf{a}, \mathbf{b}] \wedge[\mathbf{c}, \mathbf{d}]=[(\mathbf{a}+\mathbf{d}) \wedge(\mathbf{b}+\mathbf{c}), \mathbf{b}+\mathbf{d}]
$$

Furthermore, $u_{L}:=[(1),(0)]$ is a strong unit of the $\ell$-group $G_{L}$.

Remark 2.8. The standard proof of Theorem 2.7 for MV-algebras relies on the fact that if $A$ is an $\mathrm{MV}$-algebra, then $\mathbf{a} \leq \mathbf{b}$ in $M_{A}$ if and only if there exists $\mathbf{c} \in M_{A}$ such that $\mathbf{b}=\mathbf{a}+\mathbf{c}$ [3. Prop. 2.3.2]. However, this condition does not hold for general BL-algebras, not even for those of cancellative type.

Theorem 2.9. Let $L$ be a BL-algebra whose $M V$-center is denoted by $A$ with Chang-Muncidi's group $G_{A}$. Then, there exists an ideal $\mathfrak{S}(L)$ of $G_{L}$ such that

$$
G_{L}=G_{A} \oplus \mathfrak{S}(L)
$$

In particular, the Chang's $\ell$-group $G_{A}$ of $A$ is a direct summand of $G_{L}$.

Corollary 2.10. Let $L$ be a BL-algebra whose $M V$-center is A, then;

There exists an ordered-preserving group isomorphism (o-isomorphism) from $G_{L}$ onto $G_{A} \times_{l e x} \mathfrak{S}(L)$ (the lexicographic product), where $\mathfrak{S}(L)$ is the lattice ordered group above. 
For BL-algebras of cancellative type, several aspects of the construction can be simplified as we will see next.

Proposition 2.11. If $L$ is a BL-algebra of cancellative type, then $\left(M_{L},+\right)$ is a cancellative monoid, that is for every good sequences $\mathbf{a}, \mathbf{b}, \mathbf{c}$ in $L$ such that $\mathbf{a}+\mathbf{c}=\mathbf{b}+\mathbf{c}$, then $\mathbf{a}=\mathbf{b}$.

Corollary 2.12. For every BL-algebra $L$ of cancellative type, $\left(M_{L},+, \leq\right)$ is submonoid of the positive cone of $G_{L}$ and the restriction of the order of $G_{L}$ to $M_{L}$ coincides with $\leq$.

Corollary 2.13. A BL-algebra of cancellative type is an $M V$-algebra if and only if $\mathfrak{S}(L)=0$.

\section{Examples and alternate descriptions}

This section is devoted to computations of the Chang's $\ell$-group of some of the most important BLalgebras.

Remark 3.1. Suppose that $L$ is a BL-chain, then every element $[\mathbf{a}, \mathbf{b}] \in G_{L}$ has the form $\left[\left(1^{p}, a\right),\left(1^{q}, b\right)\right]$ with $p, q \geq 0, a \neq 0$ and $b \neq 0$. In addition, for a BL-chain $L$, the $\ell$-group $\mathfrak{S}(L)$ can be described as follows. $\mathfrak{S}(L)=\{[(a),(b)]: a, b \in L$ and $\overline{\bar{a}}=\overline{\bar{b}}\}=\{[(a),(b)]: a, b \in L$ and $\bar{a}=\bar{b}\}$.

Proposition 3.2. The Chang's $\ell$-group of any linearly ordered Gödel algebra is isomorphic to the o-group $\mathbb{Z}$.

Lemma 3.3. 1. If $L$ is linearly ordered $B L$-algebra, then $G_{L}$ is an o-group.

2. If $L$ is a BL-algebra of cancellative type so that $G_{L}$ is an o-group, then $L$ is a $B L$-chain.

Corollary 3.4. For every BL-chain L,

$$
G_{L} \cong G_{A} \times_{l e x} \mathfrak{S}(L)
$$

Proposition 3.5. Let $L=[0,1]$ with the product structure. Then $G_{L}$ is isomorphic to $\mathbb{Z} \times_{\text {lex }} \mathbb{R}_{+}$.

Proposition 3.6. If $L$ is a product BL-chain, then the Chang's $\ell$-group of $L$ is naturally isomorphic to $\mathbb{Z} \times_{\text {lex }} \mathfrak{H}(L)$, where $\mathfrak{H}(L)$ is the o-group whose negative cone is $L$.

Proposition 3.7. For every BL-algebra $L$ of finite order, $G_{L}=G_{M V(L)}$.

Corollary 3.8. A BL-algebra of finite order is of cancellative type if and only if it is an $M V$-algebra.

Corollary 3.9. For every Gödel-Dummit algebra $H, G_{H}$ is equal to the Chang's group of its Boolean center $M V(H)$.

As observed in Theorem 2.9 the Chang's group of a BL-algebra is built up of two essential pieces: the Chang's group of its MV-center and the ideal $\mathfrak{S}(L)$. Therefore, in order to understand the Chang's group of a general BL-algebra, it is enough to understand the group $\mathfrak{S}(L)$. To each filter $F$ of a BLalgebra $L$, one can associate an Abelian group $G_{F}$ whose elements are of the form $[a, b]$ with $a, b \in F$ and $[a, b]=[x, y]$ if and only if there exists $t \in F$ such that $a \otimes y \otimes t=x \otimes b \otimes t$. In addition $\left(G_{F},+,-, 0\right)$ is an Abelian group where:

$$
[a, b]+{ }_{G}[c, d]=[a \otimes c, b \otimes d],-[a, b]=[b, a], 0_{G}=[1,1]
$$

On the other hand, consider on $G_{F}$ the order $\leq$ defined by $[a, b] \leq[x, y]$ if and only if there exists $t \in F$ such that $a \otimes y \otimes t \leq x \otimes b \otimes t$.

Proposition 3.10. Let $L$ be a BL-algebra $L$ and $F$ a filter of $L$, then $\mathfrak{G}_{F}:=\left(G_{F},+,-, 0, \leq\right)$ is a lattice-ordered Abelian group with:

$$
[a, b] \vee[x, y]=[(a \otimes y) \vee(x \otimes b), b \otimes y] \text { and }[a, b] \wedge[x, y]=[(a \otimes y) \wedge(x \otimes b), b \otimes y]
$$


For every BL-algebra $L$, consider the set $F(L)$ of dense elements in $L$, that is:

$$
F(L)=\{x \in L: \bar{x}=0\}
$$

Then it is readily seen that $F(L)$ is a filter of $L$. For BL-algebras of cancellative type, the following result provides a direct description of the group $\mathfrak{S}(L)$ in terms of group of the filter $F(L)$.

Proposition 3.11. For every BL-algebra of cancellative type L,

$$
\mathfrak{G}_{F(L)} \cong \mathfrak{S}(L)
$$

Since there are important classes of algebras generalizing BL-algebras (e.g. MTL-algebras, residuated lattices), our future work will investigate the generalization of the constructions obtained here to these classes.

\section{References}

[1] R. Cignoli, D. Mundici, An elementary proof of Chang's completeness theorem for the infinitevalued calculus of Lukasiewicz, Studia Logica, (special issue on the treatment of uncertain information )58(1998) 79-97.

[2] R. Cignoli, D. Mundici, An elementary presentation of the equivalence between MV-algebras and $\ell$-groups with strong units, Studia Logica, special issue on Many-valued logics 61(1998) 49-64.

[3] R. Cignoli, I. D'Ottaviano, D. Mundici, Algebraic foundations of many-valued reasoning, Kluwer Academic, Dordrecht(2000).

[4] Cignoli, R., A. Torrens, An algebraic analysis of product logic, Mult.-Valued Log. 5 (2000) 45-65.

[5] C. Lele, J.B. Nganou, Algebraic Addition in BL-algebras, submitted 